\title{
Determination of element composition and extraterrestrial material occurrence in moss and lichen samples from King George Island (Antarctica) using reactor neutron activation analysis and SEM microscopy
}

\author{
Tomasz Mróz ${ }^{1}$ - Katarzyna Szufa ${ }^{2}$ - Marina V. Frontasyeva ${ }^{3}$ • Vladimir Tselmovich ${ }^{4}$. \\ Tatiana Ostrovnaya $^{3} \cdot$ Andrzej Kornaś $^{1} \cdot$ Maria A. Olech $^{5}$ - Jerzy W. Mietelski ${ }^{2}$. \\ Kamil Brudecki $^{2}$
}

Received: 20 April 2017 / Accepted: 5 October 2017 /Published online: 18 October 2017

(C) The Author(s) 2017. This article is an open access publication

\begin{abstract}
Seven lichens (Usnea antarctica and $U$. aurantiacoatra) and nine moss samples (Sanionia uncinata) collected in King George Island were analyzed using instrumental neutron activation analysis, and concentration of major and trace elements was calculated. For some elements, the concentrations observed in moss samples were higher than corresponding values reported from other sites in the Antarctica, but in the lichens, these were in the same range of concentrations. Scanning electron microscopy (SEM) and statistical analysis showed large influence of volcanic-origin particles. Also, the interplanetary cosmic particles (ICP) were observed in investigated samples, as mosses and lichens are good collectors of ICP and micrometeorites.
\end{abstract}

Responsible editor: Philippe Garrigues

Electronic supplementary material The online version of this article (https://doi.org/10.1007/s11356-017-0431-2) contains supplementary material, which is available to authorized users.

Tomasz Mróz

tmrozek@o2.pl

1 Institute of Biology, Pedagogical University of Cracow, Podchorążych 2, 30-084 Kraków, Poland

2 Henryk Niewodniczański Institute of Nuclear Physics Polish Academy of Sciences, Radzikowskiego 152, 31-342 Kraków, Poland

3 Joint Institute for Nuclear Rsearch, Joliot-Curie 6, Dubna, Russia 141980

4 Borok Geophysical Observatory, A Branch of Shmidt's Institute of Physics of the Earth of RAS, Borok, Nekouz, Yaroslavl region, Russia 152742

5 Institute of Botany, Jagiellonian University, Kopernika 27, 31-501 Kraków, Poland
Keywords Antarctica · Moss · Lichen · Biomonitoring · Space dust $\cdot$ Neutron activation analysis $\cdot$ SEM microscopy

\section{Introduction}

The Antarctic region is still the least polluted area on our planet. It is isolated by ocean, cyclonic storm belts, and hard weather conditions (Shaw 1988). However, since the arrival of first explorers in the nineteenth century and increasing human activity (scientific activity and tourism) and threaten environment of this region (Osyczka et al. 2007; Chwedorzewska and Korczak 2010), as already reported, the main sources of pollution in Antarctic area are burning of fuels and waste storing (Kabata-Pendias 2000; Bargagli 2008). According to increasing human activity, it is necessary to monitor pollution concentration and to control this unique environment. The King George Island is an example of Antarctic area with high-human activity. Ten polar stations (eight all-years and two summers only) are located on the island (Osyczka et al. 2007). They belong to Argentina, Chile, Brazil, China, Ecuador, South Korea, Peru, Poland, Russia, Uruguay, and USA. The King George Island is one of the South Shetlands Island group (Fig. 1). The mean annual temperatures are in range from -1.7 to $2.4{ }^{\circ} \mathrm{C}$, and the ice-free area of the island is about $8 \%$ (Kejna 1999; Bötler 2011). Soils on King George Island are mainly derived from volcanic rocks like basalts and andesite basalts, but in few sites also sedimentary rocks are present, and the soils formed on these rocks are cryosols, leptosols, regosols, and fluvisols. In this environment, mosses and lichens are two of the most important groups of organisms. Mosses and lichens are protecting initial soils from weather conditions and thus 
Fig. 1 Map of King George Island with sampling sites (numbers)

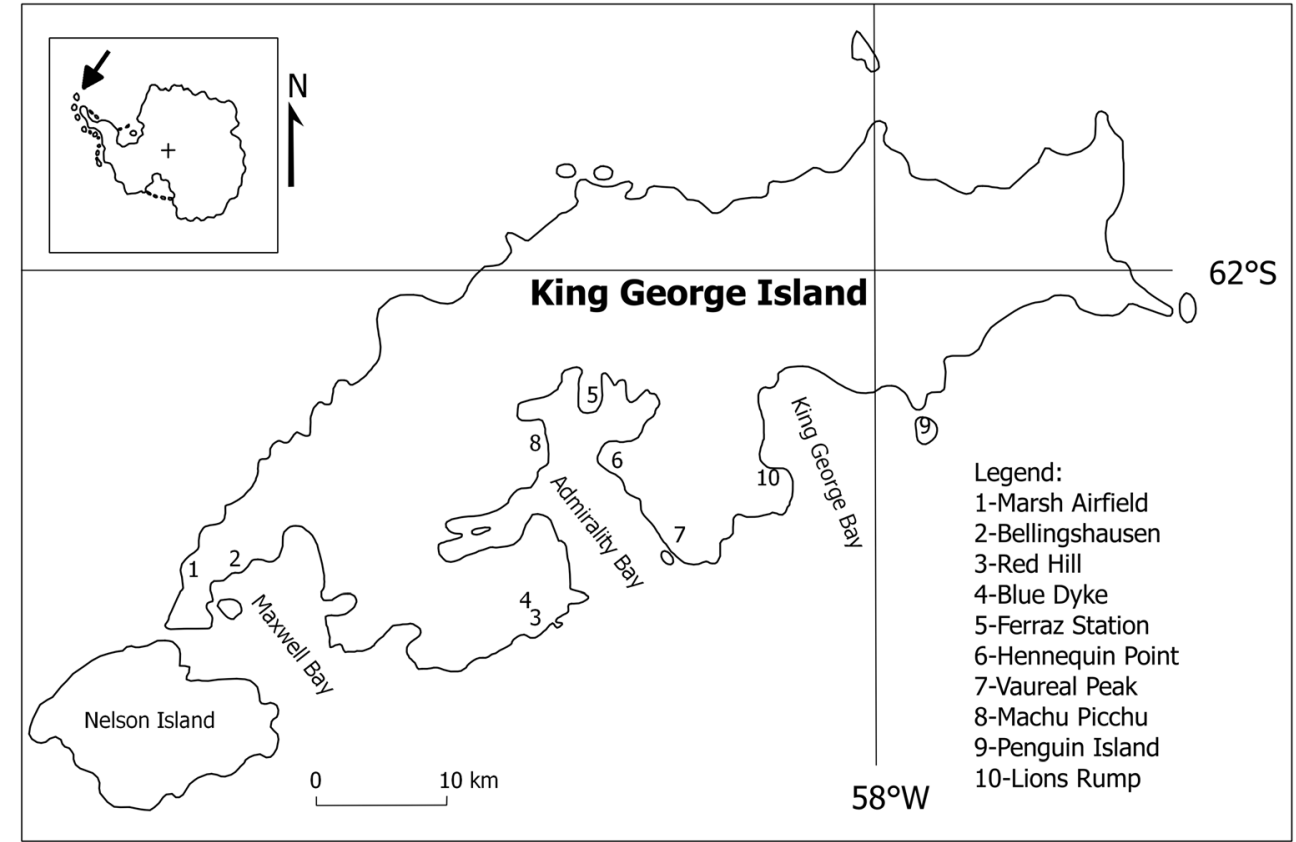

forming basic environments, which can be later populated by microbes and lower organisms (Tatur and Myrcha 1984; Bötler 2011). Since 1970s mosses and lichens are used as indicators of environmental pollution, especially air pollution (Sloof 1993; Steiness 1995; Szczepaniak and Biziuk 2003; $\mathrm{Wu}$ et al. 2014). Mosses and lichens have no roots unlike higher plants. Therefore, their basic nutrient source is atmospheric elements deposition. Because these groups of organisms have no protective cuticle, they can absorb ions from the air, rainwater, or snow. The growth rate of mosses and lichens is slow, so they can accumulate pollutants very effectively (Nash 1996; Turetsky et al. 2012). In this study, lichens Usnea antarctica (Du Rietz), U. aurantiaco-atra (Jacq.), and moss Sanionia uncinata (Hedw.) were used as bioindicators of concentration of heavy metals, rare earth elements, and trace and major elements in the air. Analysis of samples was carried out using Instrumental Neutron Activation Analysis (INAA). INAA is a non-destructive, multi-elemental method that allows simultaneous determination of about 50 elements in a single sample with mass about 300 mg (Bode 1996; Frontasyeva 2011). The advantages of INAA are low-detection limits, very precise results, and fastsample preparation without complicated chemical treatment, thus INAA is widely applied in environmental pollution studies, for example, in the Intercontinental Cooperative Programme on Effects of Air Pollution on Natural Vegetation and Crops (ICP Vegetation) research (Kłos et al. 2013; Thinova et al. 2014; Allajbeu et al. 2016; Harmens et al. 2016). The goal of this study was to estimate local environmental pollution levels and identify possible pollution sources including not only anthropogenic but also natural like, e.g., volcanic or biogenic activity, and analyze differences between trace element accumulation by mosses and lichens including investigation of rare earth elements distribution. Additionally, scanning electron microscopy (SEM) was applied to determine microparticles and presence of cosmic dust in moss and lichen samples. Cosmic dust is very abundant on earth (Brownlee 1985). The total mass of dust inside earth orbit is about $10^{15}$ tons, and every year $4 \cdot 10^{3}-10^{4}$ tons is deposited on earth surface (Yada et al. 2004; Grachev et al. 2008) that means that cosmic dust deposition should be taken account during environmental trace analysis (Yada et al. 2004). Investigations of cosmic dust were started by the HMS Challenger expedition, during which traces of cosmic matter were discovered in sea sediments (Murray and Renard 1891). Later, metallic microspherules have been found also in Antarctic and Greenland glaciers and on deserts (Maurette et al. 1986; Yada et al. 2004). Cosmic dust can be subdivided into interplanetary cosmic dust particles (IDP) or micrometeorites depending to size of particles (IDP $<30 \mu \mathrm{m}$ and micrometeorites $>50 \mu \mathrm{m}$ ), but there are no existing rigorous criteria for discrimination between cosmic dust and micrometeorites yet (Genge et al. 1997; Grachev et al. 2008). Cosmic dust particles may have very different shapes, so not only morphological criteria (spherical shape, textures, or metallic luster) but also their chemical composition is very important for confirmation of their cosmic origin. For example, volcanic-origin Fe microspheres have high-Ti content (more than 10\%) and rarely have perfect spherical shape (Szöör et al. 2001; Grachev et al. 2008). There is still no clear origin of cosmic dust. It can be remained by primordial matter from protoplanetary cloud, or it can be produced by comets and asteroids destruction (Grachev et al. 2008). 


\section{Materials and methods}

Moss and lichen samples were collected in summer seasons 2002-2003 by Polish Antarctic Expeditions of the Polish Academy of Sciences to the Henryk Arctowski Station. Sampling sites were free from direct influence of visible aerosols. Four sampling sites (1, 2, 5, and 8) were located close to polar stations. Sampling site 1 was located near Marsch Airfield, close to Chilean, Eduardo Frei base with average winter population of 80 people. Samples from sampling site number 2 were collected close to Russian Bellingshausen base with average winter population of 25 people. Sampling site number 5 was located near Brazilian station Comandante Ferraz with 12 people winter crew, and site number 8 was located close to Peruvian Macchu Picchu station (summer only station). S. uncinata (Hedw.) is one of the most common mosses in the Antarctic region (Putzke et al. 2015). It forms large area carpets, associated with other mosses and lichens. U. antarctica (Du Rietz) and $U$. aurantiacoatra (Jacq.) are lichens mostly associated with S. uncinata (Victoria et al. 2009; Victoria et al. 2013). Sampling sites (Table 1) were located within Antarctic stations operating in full-year or summer only cycle. Collected material was packed into plastic bags. After identification, samples were dried and shipped to Poland. In 2015, samples were analyzed using INAA in Frank Laboratory of Neutron Physics, Joint Institute for Nuclear Research in Dubna. Samples were once again dried in $30^{\circ} \mathrm{C}$ and cleaned mechanically from possible soil impurities using scissors and tweezers. From each sample, two portions about $300 \mathrm{mg}$ were taken to form two tablets for short and long irradiation. The mass of $300 \mathrm{mg}$ is a sufficient mass to use moss and lichen samples without homogenization (Korzewka et al. 2007; Steinnes et al. 2007). Tablets were packed in polyethylene bags and plastic containers (for short irradiation) and in aluminum foil and aluminum container for long irradiation. Samples were irradiated in IBR-2M reactor in the Frank Laboratory of Neutron Physics. For determination using short-lived isotopes $(\mathrm{Mg}, \mathrm{Al}, \mathrm{Cl}, \mathrm{Ca}, \mathrm{V}, \mathrm{Mn}, \mathrm{I}, \mathrm{Cu}, \mathrm{K}, \mathrm{S}, \mathrm{Ti}, \mathrm{In}, \mathrm{Dy}, \mathrm{Si})$, samples were irradiated for $3 \mathrm{~min}$. After short irradiation in channel 2 (without Cd screen, Table 2), samples were measured twice. First measurement was for $5 \mathrm{~min}$ after $3 \mathrm{~min}$ of decay and second one for $20 \mathrm{~min}$ after $10 \mathrm{~min}$ of decay. For determination of long-lived isotopes $(\mathrm{Na}, \mathrm{K}, \mathrm{Sc}, \mathrm{Cr}$, $\mathrm{Fe}, \mathrm{Co}, \mathrm{Ni}, \mathrm{Sb}, \mathrm{Zn}, \mathrm{As}, \mathrm{Rb}, \mathrm{Sr}, \mathrm{Cs}, \mathrm{Ba}, \mathrm{La}, \mathrm{Sm}, \mathrm{W}, \mathrm{Th}$, $\mathrm{U}, \mathrm{Cs}, \mathrm{Au}, \mathrm{Eu}, \mathrm{Nd}, \mathrm{Zr}, \mathrm{Rb}, \mathrm{Br}, \mathrm{Se}, \mathrm{Cd}, \mathrm{Ag}, \mathrm{Mo}, \mathrm{Ce}, \mathrm{Hg}, \mathrm{W}$, $\mathrm{Ta}, \mathrm{Hf}, \mathrm{Lu}, \mathrm{Yb}, \mathrm{Tm}, \mathrm{Tb}, \mathrm{Gd}$ ), samples were irradiated by $100 \mathrm{~h}$ in Cd-screened channel 1. After irradiation, samples were repacked and measured for $45 \mathrm{~min}$ and for $3 \mathrm{~h}$ after 5 and 20 days, respectively. Measurements of gamma rays were performed using High-Purity Germanium (HPGe) detector with resolution $1.9 \mathrm{keV}$ on $1332-\mathrm{keV}$ line from ${ }^{60} \mathrm{Co}$.
Data acquisition process was controlled using Genie 2000 software (Canberra). The processing of data and determination of element concentration in samples were performed using software developed in FLNP JINR (Ostrovnaya et al. 1993; Ostrovnaya 2000). For quality assurance purposes, certified reference materials (IAEA 336) and neutron flux comparators were used.

SEM microscopy and MPA analysis were performed using Tescan Vega II (Tescan, Czech Republic) with energy dispersive X-ray analyzer (EDS). Samples were analyzed with an accelerating voltage of $20 \mathrm{kV}$ and beam current $0.2 \mathrm{nA}$. Samples of mosses and lichens with mass about $300 \mathrm{mg}$ were milled in the agate mortar and sonicated. After homogenization, magnetic particles were extracted by permanent magnet. Magnetic particles collected on the magnet were transferred to double layer conductive carbon adhesive tape and placed on objective table using glass rod. Data acquisition time was 1-2 min, and obtained results were normalized to $100 \%$ (Pechersky et al. 2015a, b). Total number of 53 objects found in 14 samples of mosses and lichens were analyzed. Depending on size of object, 3 to 10 points were analyzed by EDS spectrometer.

Principal component analysis (PCA) was used for identification possible sources of elements in analyzed samples. For PCA analysis, we used Varimax rotation with Kaiser normalization (eigenvalues $>1$ ) and $p=0.05$.

Normalization of REE concentrations was done by using chondrite values following by Taylor and McLennan (1985).

\section{Results and discussion}

Tables 3 and 4 show ranges and medians for 52 element concentrations determined in moss and lichen samples. The concentrations of elements in investigated samples were different depending on sampling site and species. In general, concentrations of elements in moss samples were greater than in lichens. This observation can be explained by fact that $S$. uncinata can use rhizoids to ensure some part of water supply. Elements dissolved in soil-water may then be transported from soil to moss (Osyczka et al. 2007). Only for $\mathrm{Cl}, \mathrm{Ag}, \mathrm{I}, \mathrm{Lu}$, and $\mathrm{W}$ concentrations were greater in lichens than in mosses. The excess of $\mathrm{I}$ and $\mathrm{Cl}$ in lichens as compared to mosses can be probably an effect of organohalogen synthesis by fungal part of lichen (Matschullat et al. 1999; Gribble 2010).

\section{Rare earth elements}

In this group, concentrations of 12 elements $(\mathrm{Sc}, \mathrm{La}, \mathrm{Ce}$, $\mathrm{Nd}, \mathrm{Sm}, \mathrm{Eu}, \mathrm{Gd}, \mathrm{Tb}, \mathrm{Dy}, \mathrm{Tm}, \mathrm{Yb}, \mathrm{Lu}$ ) were measured. Rare earth elements (REE) are naturally occurring ingredients of lithosphere commonly used for tracing geochemical 
Table 1 Sample description

\begin{tabular}{|c|c|c|c|c|}
\hline No. & Species & Location & Coordinates & Sample code \\
\hline \multicolumn{5}{|c|}{ Lichens } \\
\hline 1 & Usnea antarctica & Marsh Airfield & $\begin{array}{l}62^{\circ} 11^{\prime} 39,5^{\prime \prime} \mathrm{S} \\
58^{\circ} 58^{\prime} 35,0^{\prime \prime} \mathrm{W}\end{array}$ & $\mathrm{i}-01$ \\
\hline 2 & Usnea antarctica & Red Hill & $\begin{array}{l}62^{\circ} 13^{\prime} 59,98^{\prime \prime} \mathrm{S} \\
58^{\circ} 30^{\prime} 00^{\prime \prime} \mathrm{W}\end{array}$ & $\mathrm{i}-02$ \\
\hline 3 & Usnea antarctica & Ferraz Station & $\begin{array}{l}62^{\circ} 05^{\prime} 00^{\prime \prime} \mathrm{S} \\
58^{\circ} 23^{\prime} 28^{\prime \prime} \mathrm{W}\end{array}$ & $\mathrm{i}-03$ \\
\hline 4 & Usnea aurantiaco-atra & Bellingshausen & $\begin{array}{l}62^{\circ} 12^{\prime} \mathrm{S} \\
58^{\circ} 58^{\prime} \mathrm{W}\end{array}$ & $\mathrm{i}-04$ \\
\hline 5 & Usnea aurantiaco-atra & Hennequin Point & $\begin{array}{l}62^{\circ} 07^{\prime} 13,0^{\prime \prime} \mathrm{S} \\
58^{\circ} 23^{\prime} 46,4^{\prime \prime} \mathrm{W}\end{array}$ & $\mathrm{i}-05$ \\
\hline 6 & Usnea antarctica & Penguin Island & $\begin{array}{l}62^{\circ} 06^{\prime} 15,6^{\prime \prime} \mathrm{S} \\
57^{\circ} 59^{\prime} 40,2^{\prime \prime} \mathrm{W}\end{array}$ & $\mathrm{i}-06$ \\
\hline 7 & Usnea antarctica & Vaureal Peak & $\begin{array}{l}62^{\circ} 10^{\prime} 58,2^{\prime \prime} \mathrm{S} \\
58^{\circ} 17^{\prime} 32,3^{\prime \prime} \mathrm{W}\end{array}$ & $\mathrm{i}-07$ \\
\hline \multicolumn{5}{|c|}{ Mosses } \\
\hline 1 & Sanonia uncinata & Hennequin Point & $\begin{array}{l}62^{\circ} 07^{\prime} 13,0^{\prime \prime} \mathrm{S} \\
58^{\circ} 23^{\prime} 46.4^{\prime \prime} \mathrm{W}\end{array}$ & $\mathrm{j}-01$ \\
\hline 2 & Sanonia uncinata & Marsh Airfield & $\begin{array}{l}62^{\circ} 11^{\prime} 18,5^{\prime \prime} \mathrm{S} \\
58^{\circ} 59^{\prime} 56.2^{\prime \prime} \mathrm{W}\end{array}$ & $\mathrm{j}-02$ \\
\hline 3 & Sanonia uncinata & Bellingshausen & $\begin{array}{l}62^{\circ} 12^{\prime} \mathrm{S} \\
58^{\circ} 58^{\prime} \mathrm{W}\end{array}$ & $\mathrm{j}-03$ \\
\hline 4 & Sanonia uncinata & Machu Picchu & $\begin{array}{l}62^{\circ} 05^{\prime} 30^{\prime \prime} \mathrm{S} \\
58^{\circ} 28^{\prime} 14^{\prime \prime} \mathrm{W}\end{array}$ & $\mathrm{j}-04$ \\
\hline 5 & Sanonia uncinata & Blue Dyke & $\begin{array}{l}62^{\circ} 13^{\prime} 30^{\prime \prime} \mathrm{S} \\
58^{\circ} 28^{\prime} 14^{\prime \prime} \mathrm{W}\end{array}$ & $\mathrm{j}-05$ \\
\hline 6 & Sanonia uncinata & Vaureal Peak & $\begin{array}{l}62^{\circ} 10^{\prime} 52,8^{\prime \prime} \mathrm{S} \\
58^{\circ} 17^{\prime} 32,3^{\prime \prime} \mathrm{W}\end{array}$ & $\mathrm{j}-06$ \\
\hline 7 & Sanonia uncinata & Ferraz Station & $\begin{array}{l}62^{\circ} 08^{\prime} 65^{\prime \prime} \mathrm{S} \\
58^{\circ} 39^{\prime} 32^{\prime \prime} \mathrm{W}\end{array}$ & $\mathrm{j}-07$ \\
\hline 8 & Sanonia uncinata & Penguin Island & $\begin{array}{l}62^{\circ} 06^{\prime} \mathrm{S} \\
57^{\circ} 56^{\prime} \mathrm{W}\end{array}$ & $\mathrm{j}-09$ \\
\hline 9 & Sanonia uncinata & Lions Rump & $\begin{array}{l}62^{\circ} 08^{\prime} 01^{\prime \prime} \mathrm{S} \\
58^{\circ} 07^{\prime} 25^{\prime \prime} \mathrm{W}\end{array}$ & $\mathrm{j}-10$ \\
\hline
\end{tabular}

processes, but also are widely applied in industry (e.g., electronics or medicine), and therefore anthropogenic contamination with REE is possible (Brioschi et al. 2013; Allajbeu et al. 2016). For lichens, the highest concentrations of REE were usually found on Red Hill and the lowest on Varueal Peak. In case of mosses, the highest concentrations were measured in samples collected near Ferraz Station and the lowest in samples from Blue Dyke. For RRE, chondrite normalization (Taylor and McLennan
1985) was applied and geochemical parameters were calculated to obtain data about their fractionation, and results are showed in Table 5.

In Table 5, upper continental crust (UCC) is a reference composition (Rudnick and Gao 2004). Values of all REE concentrations are lower than reference values for UCC. For geochemical parameters, there were no significant differences between mosses and lichens, except La/Th and $\mathrm{Tm} / \mathrm{Tb}$ ratios, but in these cases, standard deviation in
Table 2 Neutron flux parameters of irradiation channels

\begin{tabular}{lllll}
\hline Irradiation channel & $\begin{array}{l}\Phi \cdot 10^{12}\left[n \cdot \mathrm{cm}^{-2} \cdot \mathrm{s}^{-1}\right] \\
0<E<0.55[\mathrm{eV}]\end{array}$ & $\begin{array}{l}\Phi \cdot 10^{12}\left[n \cdot \mathrm{cm}^{-2} \cdot \mathrm{s}^{-1}\right] \\
0.55<E<10^{5}[\mathrm{eV}]\end{array}$ & $\begin{array}{l}\Phi \cdot 10^{12}\left[n \cdot \mathrm{cm}^{-2} \cdot \mathrm{s}^{-1}\right] \\
10^{5}<E<25 \cdot 10^{6}[\mathrm{eV}]\end{array}$ & $T\left({ }^{\circ} \mathrm{C}\right)$ \\
& Thermal & Fast & \\
\hline Ch1 (Cd-screened) & 0.02 & 3.3 & 4.2 & 70 \\
Ch2 & 1.2 & 3.0 & 4.1 & 60 \\
\hline
\end{tabular}


Table 3 Results of lichens samples analysis

Elements concentrations in lichens samples $(n=7)$

\begin{tabular}{|c|c|c|}
\hline & Median (mg/kg) & Range (mg/kg) \\
\hline $\mathrm{Na}$ & $638 \pm 19$ & $435 \pm 13 \div 8040 \pm 240$ \\
\hline $\mathrm{Mg}$ & $794 \pm 32$ & $395 \pm 16 \div 2060 \pm 62$ \\
\hline $\mathrm{Al}$ & $832 \pm 25$ & $246 \pm 7 \div 2900 \pm 87$ \\
\hline $\mathrm{Si}$ & $12,300 \pm 3700$ & $8300 \pm 2500 \div 24,400 \pm 7300$ \\
\hline S & $5000 \pm 1500$ & $3410 \pm 1000 \div 5940 \pm 1800$ \\
\hline $\mathrm{Cl}$ & $1146 \pm 92$ & $426 \pm 34 \div 15,600 \pm 1200$ \\
\hline $\mathrm{K}$ & $2070 \pm 210$ & $1650 \pm 170 \div 2750 \pm 300$ \\
\hline $\mathrm{Ca}$ & $8970 \pm 630$ & $1530 \pm 120 \div 14,900 \pm 1000$ \\
\hline $\mathrm{Sc}$ & $0.76 \pm 0.02$ & $0.32 \pm 0.01 \div 4.01 \pm 0.12$ \\
\hline $\mathrm{Ti}$ & $110 \pm 8$ & $25.7 \pm 5.4 \div 241 \pm 15$ \\
\hline $\mathrm{V}$ & $1.95 \pm 0.09$ & $0.59 \pm 0.04 \div 9.1 \pm 0.3$ \\
\hline $\mathrm{Cr}$ & $2.99 \pm 0.90$ & $0.701 \pm 0.210 \div 4.02 \pm 0.68$ \\
\hline $\mathrm{Mn}$ & $14.2 \pm 0.9$ & $10.1 \pm 0.7 \div 35.8 \pm 2.2$ \\
\hline $\mathrm{Fe}$ & $441 \pm 35$ & $126 \pm 14 \div 7140 \pm 430$ \\
\hline $\mathrm{Ni}$ & $2.02 \pm 0.61$ & $1.43 \pm 0.43 \div 3.48 \pm 0.14$ \\
\hline Co & $0.208 \pm 0.023$ & $0.091 \pm 0.013 \div 3.23 \pm 0.26$ \\
\hline $\mathrm{Cu}$ & $12.7 \pm 3.8$ & $6.6 \pm 2.0 \div 29.3 \pm 8.8$ \\
\hline $\mathrm{Zn}$ & $14.0 \pm 0.7$ & $5.6 \pm 0.3 \div 33.9 \pm 1$ \\
\hline $\mathrm{Se}$ & $0.759 \pm 0.053$ & $0.428 \pm 0.034 \div 1.05 \pm 0.07$ \\
\hline As & $0.261 \pm 0.010$ & $0.21 \pm 0.01 \div 0.578 \pm 0.023$ \\
\hline $\mathrm{Br}$ & $41.9 \pm 1.3$ & $15.9 \pm 0.5 \div 88.9 \pm 2.7$ \\
\hline $\mathrm{Rb}$ & $1.04 \pm 0.18$ & $0.78 \pm 0.14 \div 3.76 \pm 0.64$ \\
\hline $\mathrm{Sr}$ & $30.5 \pm 2.8$ & $13.7 \pm 1.4 \div 260 \pm 23$ \\
\hline $\mathrm{Zr}$ & $4.61 \pm 1.38$ & $3.8 \pm 1.1 \div 9.2 \pm 2.8$ \\
\hline Mo & $0.145 \pm 0.044$ & $0.113 \pm 0.034 \div 0.235 \pm 0.071$ \\
\hline $\mathrm{Ag}$ & $0.79 \pm 0.24$ & $0.057 \pm 0.017 \div 0.138 \pm 0.041$ \\
\hline $\mathrm{Cd}$ & $<\mathrm{MDC}$ & $<\mathrm{MDC}$ \\
\hline In & $0.070 \pm 0.022$ & $0.019 \pm 0.007 \div 0.139 \pm 0.046$ \\
\hline $\mathrm{Sb}$ & $0.0124 \pm 0.0025$ & $0.004 \pm 0.002 \div 0.0413 \pm 0.005$ \\
\hline I & $5.5 \pm 2.1$ & $1.76 \pm 0.67 \div 7.62 \pm 2.89$ \\
\hline $\mathrm{Ba}$ & $2.05 \pm 0.33$ & $0.78 \pm 0.27 \div 18.2 \pm 1.1$ \\
\hline Cs & $0.023 \pm 0.004$ & $0.008 \pm 0.001 \div 0.08 \pm 0.01$ \\
\hline $\mathrm{La}$ & $2.03 \pm 0.08$ & $0.46 \pm 0.02 \div 4.65 \pm 0.14$ \\
\hline $\mathrm{Ce}$ & $4.26 \pm 0.29$ & $1.12 \pm 0.15 \div 9.84 \pm 0.59$ \\
\hline $\mathrm{Nd}$ & $2.96 \pm 0.72$ & $0.75 \pm 0.23 \div 6.9 \pm 2.2$ \\
\hline $\mathrm{Sm}$ & $0.628 \pm 0.031$ & $0.127 \pm 0.007 \div 1.67 \pm 0.08$ \\
\hline $\mathrm{Eu}$ & $0.115 \pm 0.016$ & $0.0218 \pm 0.0075 \div 0.448 \pm 0.031$ \\
\hline $\mathrm{Gd}$ & $0.126 \pm 0.021$ & $0.062 \pm 0.019 \div 0.478 \pm 0.038$ \\
\hline $\mathrm{Tb}$ & $0.0793 \pm 0.0024$ & $0.0165 \pm 0.0008 \div 0.231 \pm 0.005$ \\
\hline Dy & $0.420 \pm 0.15$ & $0.054 \pm 0.019 \div 1.11 \pm 0.39$ \\
\hline $\mathrm{Tm}$ & $0.0320 \pm 0.0096$ & $0.025 \pm 0.008 \div 0.114 \pm 0.003$ \\
\hline $\mathrm{Yb}$ & $0.208 \pm 0.023$ & $0.063 \pm 0.016 \div 0.669 \pm 0.047$ \\
\hline $\mathrm{Lu}$ & $0.144 \pm 0.043$ & $0.081 \pm 0.024 \div 0.218 \pm 0.065$ \\
\hline Hf & $0.049 \pm 0.015$ & $0.015 \pm 0.006 \div 0.197 \pm 0.061$ \\
\hline $\mathrm{Ta}$ & $0.0057 \pm 0.0017$ & $0.0036 \pm 0.0011 \div 0.0137 \pm 0.0009$ \\
\hline $\mathrm{W}$ & $0.163 \pm 0.049$ & $0.105 \pm 0.032 \div 0.275 \pm 0.083$ \\
\hline $\mathrm{Au}$ & $0.00036 \pm 0.00012$ & $0.00012 \pm 0.00005 \div 0.00064 \pm 0.00021$ \\
\hline $\mathrm{Hg}$ & $0.101 \pm 0.030$ & $0.0694 \pm 0.0208 \div 0.117 \pm 0.035$ \\
\hline $\mathrm{Th}$ & $0.103 \pm 0.006$ & $0.0369 \pm 0.0026 \div 0.249 \pm 0.013$ \\
\hline $\mathrm{U}$ & $0.0392 \pm 0.0035$ & $0.0202 \pm 0.0030 \div 0.0767 \pm 0.0046$ \\
\hline
\end{tabular}

Table 4 Results of moss samples analysis

Element concentrations in moss samples $(n=9)$

\begin{tabular}{|c|c|c|}
\hline & Median (mg/kg) & Range (mg/kg) \\
\hline $\mathrm{Na}$ & $8010 \pm 24$ & $4210 \pm 126 \div 11,300 \pm 340$ \\
\hline $\mathrm{Mg}$ & $17,300 \pm 520$ & $6790 \pm 204 \div 28,600 \pm 570$ \\
\hline $\mathrm{Al}$ & $26,800 \pm 610$ & $12,400 \pm 250 \div 50,200 \pm 1000$ \\
\hline $\mathrm{Si}$ & $59,000 \pm 14,000$ & $16,100 \pm 5600 \div 105,000 \pm 29,000$ \\
\hline $\mathrm{S}$ & $27,100 \pm 8100$ & $13,700 \pm 4110 \div 46,000 \pm 14,000$ \\
\hline $\mathrm{Cl}$ & $512 \pm 44$ & $243 \pm 24 \div 3700 \pm 300$ \\
\hline $\mathrm{K}$ & $4970 \pm 500$ & $3300 \pm 330 \div 8260 \pm 740$ \\
\hline $\mathrm{Ca}$ & $15,900 \pm 950$ & $7540 \pm 680 \div 23,600 \pm 1200$ \\
\hline $\mathrm{Sc}$ & $9.45 \pm 0.19$ & $4.20 \pm 0.13 \div 15.7 \pm 0.3$ \\
\hline $\mathrm{Ti}$ & $1650 \pm 120$ & $765 \pm 46 \div 2700 \pm 160$ \\
\hline $\mathrm{V}$ & $73.2 \pm 1.5$ & $29.4 \pm 0.9 \div 118 \pm 4$ \\
\hline $\mathrm{Cr}$ & $40.0 \pm 2.4$ & $4.48 \pm 0.99 \div 153 \pm 5$ \\
\hline $\mathrm{Mn}$ & $341 \pm 21$ & $168 \pm 10 \div 598 \pm 36$ \\
\hline $\mathrm{Fe}$ & $22,500 \pm 1100$ & $6830 \pm 410 \div 39,500 \pm 2000$ \\
\hline $\mathrm{Ni}$ & $8.51 \pm 1.53$ & $3.99 \pm 1.08 \div 65.9 \pm 5.3$ \\
\hline $\mathrm{Co}$ & $12.4 \pm 0.9$ & $4.06 \pm 0.33 \div 19.2 \pm 1.5$ \\
\hline $\mathrm{Cu}$ & $53.6 \pm 16.1$ & $29.6 \pm 8.9 \div 96.7 \pm 29.1$ \\
\hline $\mathrm{Zn}$ & $31.2 \pm 0.6$ & $20.0 \pm 0.4 \div 62.2 \pm 1.9$ \\
\hline $\mathrm{Se}$ & $1.14 \pm 0.08$ & $0.515 \pm 0.052 \div 8.43 \pm 0.42$ \\
\hline As & $1.30 \pm 0.09$ & $0.363 \pm 0.022 \div 7.77 \pm 0.39$ \\
\hline $\mathrm{Br}$ & $67.9 \pm 2.1$ & $24.4 \pm 0.7 \div 133 \pm 4$ \\
\hline $\mathrm{Rb}$ & $6.52 \pm 1.04$ & $2.60 \pm 0.44 \div 16.8 \pm 2.7$ \\
\hline $\mathrm{Sr}$ & $167 \pm 15$ & $101 \pm 9 \div 278 \pm 25$ \\
\hline $\mathrm{Zr}$ & $29.7 \pm 9.8$ & $9.41 \pm 3.39 \div 87.8 \pm 27.2$ \\
\hline Mo & $0.548 \pm 0.181$ & $0.144 \pm 0.061 \div 4.27 \pm 14.09$ \\
\hline $\mathrm{Ag}$ & $0.133 \pm 0.024$ & $0.0552 \pm 0.0049 \div 0.318 \pm 0.032$ \\
\hline $\mathrm{Cd}$ & $1.04 \pm 0.31$ & $0.204 \pm 0.084 \div 1.41 \pm 0.42$ \\
\hline In & $0.121 \pm 0.038$ & $0.0981 \pm 0.0304 \div 0.144 \pm 0.045$ \\
\hline $\mathrm{Sb}$ & $0.0557 \pm 0.0067$ & $0.0261 \pm 0.0029 \div 0.259 \pm 0.026$ \\
\hline I & $3.42 \pm 1.23$ & $0.85 \pm 0.26 \div 4.51 \pm 1.58$ \\
\hline $\mathrm{Ba}$ & $74.9 \pm 3.8$ & $28.7 \pm 1.7 \div 189 \pm 10$ \\
\hline $\mathrm{Cs}$ & $0.363 \pm 0.015$ & $0.0906 \pm 0.0073 \div 1.31 \pm 0.04$ \\
\hline $\mathrm{La}$ & $5.24 \pm 0.21$ & $2.39 \pm 0.09 \div 13.3 \pm 0.4$ \\
\hline $\mathrm{Ce}$ & $11.6 \pm 0.8$ & $7.86 \pm 0.63 \div 26.8 \pm 1.6$ \\
\hline $\mathrm{Nd}$ & $6.75 \pm 2.23$ & $1.46 \pm 0.43 \div 20.5 \pm 6.8$ \\
\hline $\mathrm{Sm}$ & $1.44 \pm 0.09$ & $0.818 \pm 0.057 \div 2.15 \pm 0.15$ \\
\hline $\mathrm{Eu}$ & $0.563 \pm 0.034$ & $0.321 \pm 0.032 \div 0.862 \pm 0.051$ \\
\hline Gd & $0.510 \pm 0.051$ & $0.180 \pm 0.023 \div 1.77 \pm 0.14$ \\
\hline $\mathrm{Tb}$ & $0.212 \pm 0.004$ & $0.154 \pm 0.005 \div 0.371 \pm 0.007$ \\
\hline Dy & $0.937 \pm 0.375$ & $0.649 \pm 0.241 \div 1.77 \pm 0.64$ \\
\hline $\mathrm{Tm}$ & $0.099 \pm 0.011$ & $0.0638 \pm 0.0071 \div 0.161 \pm 0.016$ \\
\hline $\mathrm{Yb}$ & $0.624 \pm 0.056$ & $0.492 \pm 0.049 \div 1.08 \pm 0.09$ \\
\hline $\mathrm{Lu}$ & $0.089 \pm 0.027$ & $0.0274 \pm 0.0082 \div 0.125 \pm 0.038$ \\
\hline $\mathrm{Hf}$ & $1.06 \pm 0.32$ & $0.61 \pm 0.18 \div 2.82 \pm 0.85$ \\
\hline $\mathrm{Ta}$ & $0.0515 \pm 0.0015$ & $0.0404 \pm 0.0024 \div 0.113 \pm 0.003$ \\
\hline $\mathrm{W}$ & $0.095 \pm 0.028$ & $0.0299 \pm 0.0089 \div 0.212 \pm 0.069$ \\
\hline $\mathrm{Au}$ & $0.0057 \pm 0.0018$ & $0.00091 \pm 0.00052 \div 0.0154 \pm 0.0046$ \\
\hline $\mathrm{Hg}$ & $0.68 \pm 0.20$ & $0.56 \pm 0.17 \div 1.06 \pm 0.32$ \\
\hline Th & $0.975 \pm 0.049$ & $0.192 \pm 0.012 \div 2.84 \pm 0.14$ \\
\hline $\mathrm{U}$ & $0.465 \pm 0.023$ & $0.133 \pm 0.008 \div 1.67 \pm 0.08$ \\
\hline
\end{tabular}


Table 5 Geochemical parameters for rare earth elements (RRE) and chondrite normalized concentrations in samples and upper continental crust (UCC)

\begin{tabular}{lccc}
\hline Element & Lichens normalized $(\mathrm{mg} / \mathrm{kg})$ & Mosses normalized $(\mathrm{mg} / \mathrm{kg})$ & UCC normalized $(\mathrm{mg} / \mathrm{kg})$ \\
\hline $\mathrm{La}$ & $5.45 \pm 3.62$ & $18.15 \pm 10.16$ & 84.47 \\
$\mathrm{Ce}$ & $4.32 \pm 2.91$ & $14.11 \pm 7.53$ & 65.83 \\
$\mathrm{Nd}$ & $3.95 \pm 2.70$ & $12.37 \pm 7.74$ & 37.97 \\
$\mathrm{Sm}$ & $2.78 \pm 2.15$ & $7.19 \pm 3.51$ & 20.35 \\
$\mathrm{Eu}$ & $1.79 \pm 1.52$ & $6.50 \pm 2.24$ & 11.49 \\
$\mathrm{Gd}$ & $0.56 \pm 0.45$ & $2.04 \pm 1.61$ & 13.07 \\
$\mathrm{~Tb}$ & $1.54 \pm 1.20$ & $4.21 \pm 1.51$ & 12.07 \\
$\mathrm{Dy}$ & $1.21 \pm 0.90$ & $3.13 \pm 1.37$ & 10.24 \\
$\mathrm{Tm}$ & $1.28 \pm 0.82$ & $2.89 \pm 0.90$ & 8.22 \\
$\mathrm{Yb}$ & $1.03 \pm 0.80$ & $2.96 \pm 0.96$ & 8.06 \\
$\mathrm{Lu}$ & $3.83 \pm 1.30$ & $2.45 \pm 0.80$ & 8.14 \\
$\mathrm{Geochemical}$ & parameters* & & \\
$(\mathrm{Ce} / \mathrm{Yb}) n$ & $4.53 \pm 1.08$ & $4.73 \pm 1.93$ & 8.16 \\
$(\mathrm{Gd} / \mathrm{Yb}) \mathrm{n}$ & $0.60 \pm 0.19$ & $0.64 \pm 0.35$ & 1.62 \\
$(\mathrm{La} / \mathrm{Sm}) \mathrm{n}$ & $2.17 \pm 0.53$ & $2.47 \pm 0.38$ & 4.15 \\
$\delta \mathrm{Eu}$ & $1.48 \pm 0.59$ & $1.98 \pm 0.61$ & 0.70 \\
$(\mathrm{La} / \mathrm{Yb}) n$ & $5.80 \pm 1.92$ & $6.05 \pm 2.65$ & 10.47 \\
$\mathrm{Th} / \mathrm{Sc}$ & $0.11 \pm 0.04$ & $0.10 \pm 0.07$ & 0.75 \\
$\mathrm{La} / \mathrm{Th}$ & $20.86 \pm 11.97$ & $7.82 \pm 2.93$ & 2.95 \\
$\mathrm{Sm} / \mathrm{La}$ & $0.31 \pm 0.07$ & $0.26 \pm 0.04$ & 0.15 \\
$\mathrm{Tm} / \mathrm{Tb}$ & $0.73 \pm 0.50$ & $0.44 \pm 0.07$ & 0.43 \\
\hline $\mathrm{See}$ & &
\end{tabular}

-See text for details lichens was high. That means that both groups of organisms are accumulating REE similarly. Both groups show fractionation of REE. For example, $\delta \mathrm{Eu}$ is more than two times higher than reference value for UCC. Eu anomalies

Table 6 Concentrations of heavy metals and Se compared with literature data, reference plant (RP), and Norway moss survey (Bargagli et al. 1999; Bargagli et al. 2000; Gonzáles et al. 2002; Ganeva and Yurukova 2004; Smykla et al. 2005; Barandovski et al. 2006; Osycza et al. 2007; Lim et al. 2009; Mão de Ferro et al. 2013; Zvěřina et al. 2014; Amaro et al. 2015; Bubach et al. 2016)

\begin{tabular}{|c|c|c|c|c|c|c|}
\hline Element & $\begin{array}{l}\text { Moss median } \\
(\mathrm{mg} / \mathrm{kg})\end{array}$ & $\begin{array}{l}\text { Literature range } \\
\text { for moss }(\mathrm{mg} / \mathrm{kg})\end{array}$ & $\begin{array}{l}\text { Lichen median } \\
(\mathrm{mg} / \mathrm{kg})\end{array}$ & $\begin{array}{l}\text { Literature range } \\
\text { for lichen }(\mathrm{mg} / \mathrm{kg})\end{array}$ & $\begin{array}{l}\text { Norway } \\
(\mathrm{mg} / \mathrm{kg})\end{array}$ & $\mathrm{RP}(\mathrm{mg} / \mathrm{kg})$ \\
\hline $\mathrm{V}$ & $73.2 \pm 1.5$ & $5 \div 75$ & $1.95 \pm 0.09$ & $1 \div 75$ & 0.92 & 0.50 \\
\hline $\mathrm{Cr}$ & $40.0 \pm 2.4$ & $4 \div 9$ & $2.99 \pm 0.90$ & $0.02 \div 6.8$ & 0.55 & 1.50 \\
\hline $\mathrm{Mn}$ & $341 \pm 21$ & $68 \div 390$ & $14.2 \pm 0.9$ & $10 \div 180$ & 256 & 200 \\
\hline $\mathrm{Fe}$ & $22,500 \pm 1125$ & $3500 \div 21,800$ & $441 \pm 35$ & $205 \div 12,670$ & 209 & 150 \\
\hline $\mathrm{Ni}$ & $8.51 \pm 1.53$ & $1.5 \div 2.8$ & $2.02 \pm 0.61$ & $1 \div 5.1$ & 1.14 & 1.50 \\
\hline Co & $12.4 \pm 0.9$ & $2.6 \div 2.7$ & $0.208 \pm 0.023$ & $0.02 \div 1.6$ & 0.202 & 0.20 \\
\hline $\mathrm{Cu}$ & $53.6 \pm 16.1$ & $2 \div 121$ & $12.7 \pm 3.8$ & $1.8 \div 6.7$ & 3.6 & 10 \\
\hline $\mathrm{Zn}$ & $31.2 \pm 0.6$ & $6 \div 67$ & $14.0 \pm 0.7$ & $0.59 \div 71.9$ & 26.5 & 50 \\
\hline $\mathrm{Se}$ & $1.14 \pm 0.08$ & $0.5 \div 0.6$ & $0.759 \pm 0.053$ & $0.1 \div 0.3$ & 0.093 & 0.02 \\
\hline As & $1.30 \pm 0.09$ & $0.7 \div 38$ & $0.261 \pm 0.010$ & $0.11 \div 2.3$ & 0.33 & 0.1 \\
\hline Mo & $0.548 \pm 0.181$ & $0.2 \div 0.7$ & $0.145 \pm 0.044$ & - & 0.135 & 0.5 \\
\hline $\mathrm{Cd}$ & $1.04 \pm 0.31$ & $0.05 \div 0.92$ & $<\mathrm{MDC}$ & $0.01 \div 0.05$ & 0.058 & 0.05 \\
\hline $\mathrm{Sb}$ & $0.0557 \pm 0.0067$ & $0.03 \div 0.13$ & $0.0124 \pm 0.0025$ & - & 0.033 & 0.1 \\
\hline W & $0.0946 \pm 0.0284$ & - & $0.163 \pm 0.049$ & - & 0.127 & 0.2 \\
\hline $\mathrm{Hg}$ & $0.675 \pm 0.203$ & $0.055 \div 0.56$ & $0.101 \pm 0.030$ & $0.026 \div 0.190$ & 0.046 & 0.1 \\
\hline
\end{tabular}


were calculated using equation proposed by Taylor and McLennan (1995):

$\delta \mathrm{Eu}=\frac{\mathrm{Eu}_{\mathrm{n}}}{\sqrt{\left([\mathrm{Sm}]_{\mathrm{n}} \times[\mathrm{Gd}]_{\mathrm{n}}\right)}}$

where $\mathrm{Eu}_{\mathrm{n}},[\mathrm{Sm}]_{\mathrm{n}}$, and $[\mathrm{Gd}]_{\mathrm{n}}$ are chondrite normalized concentrations of europium, samarium, and gadolinium. Similar observations were reported in other studies (Ryghaug 1983; Aubert et al. 2006) and show positive anomaly for heavy REE (Allajbeu et al. 2016). All of calculated parameters show REE distribution patterns different than UCC. The La/Th ratio higher than UCC can be evidence for sedimentary rockoriginated REE's deposited on mosses and lichens (McLennan et al. 1993).

\section{Heavy metals}

To asses King George contamination, 15 heavy metals (V, $\mathrm{Cr}, \mathrm{Mn}, \mathrm{Fe}, \mathrm{Ni}, \mathrm{Co}, \mathrm{Cu}, \mathrm{Zn}, \mathrm{Se}, \mathrm{As}, \mathrm{Mo}, \mathrm{Cd}, \mathrm{Sb}, \mathrm{W}$, and $\mathrm{Hg}$ ) were measured. For lichen samples, most contaminated area was Marsh Airfield. Only three elements $(\mathrm{Cd}$, Mo, and Se) had maximal concentrations on Red Hill. In moss samples, it was difficult to choose one most contaminated site. Highest concentrations of $\mathrm{Mn}, \mathrm{Fe}, \mathrm{Co}, \mathrm{Zn}, \mathrm{Sb}$, and V were founded near Bellingshausen station. Marsh Airfield had the highest $\mathrm{Cd}$ concentration. Maximal concentrations of Mo, As, and Se were measured in samples collected near Machu Picchu base. Varueal Peak was a sampling site with highest $\mathrm{Cu}$ concentration and Lions Ramp with $\mathrm{Cr}$ and $\mathrm{Ni}$. Median values of heavy metal concentration in lichen and moss samples from King George Island can be compared with values from Norway (Steinnes 2005; Barandovski et al. 2006), reference plant model (Markert 1991), and literature data for Antarctic region (Bargagli et al. 1999; Bargagli et al. 2000; Gonzáles et al. 2002; Smykla et al. 2005; Lim et al. 2009; Osyczka et al. 2007; Zvěřina et al. 2014; Bubach et al. 2016) (Tables 6 and 7).

Obtained results are general in the range of literature data. In moss samples, however, concentrations of $\mathrm{Cr}$ and $\mathrm{Ni}$ are elevated. The same situation can be observed for Se in lichen samples. In comparison to values from Norway, concentrations of almost all heavy metals are elevated; only depositions of $\mathrm{W}$ and $\mathrm{Zn}$ are lower.

\section{Sources of pollutants}

There are many possible sources of contamination of investigated area. The possible sources can be elevated natural background, local contamination from human activity, or longrange atmospheric transport. It was reported that As from Chilean $\mathrm{Cu}$ mines was found in Antarctic ice cores. Also, important source of As and Se is combustion of coal with
Table 7 Factor analysis of NAA data on moss and lichen samples (Rotation method: Varimax with Kaiser normalization)

\begin{tabular}{|c|c|c|c|c|}
\hline \multirow[t]{2}{*}{ Element } & \multicolumn{4}{|c|}{ Factor number } \\
\hline & 1 & 2 & 3 & 4 \\
\hline $\mathrm{Na}$ & 0.597 & 0.670 & -0.072 & 0.135 \\
\hline $\mathrm{Mg}$ & 0.302 & 0.917 & -0.008 & 0.021 \\
\hline $\mathrm{Al}$ & 0.573 & 0.772 & 0.022 & 0.210 \\
\hline $\mathrm{Si}$ & 0.512 & 0.690 & 0.023 & 0.427 \\
\hline $\mathrm{S}$ & 0.442 & 0.833 & 0.057 & -0.064 \\
\hline $\mathrm{Cl}$ & -0.125 & -0.142 & -0.107 & -0.085 \\
\hline $\mathrm{K}$ & 0.736 & 0.280 & 0.139 & 0.359 \\
\hline $\mathrm{Ca}$ & 0.093 & 0.791 & 0.434 & 0.134 \\
\hline $\mathrm{Sc}$ & 0.497 & 0.828 & -0.047 & 0.037 \\
\hline $\mathrm{Ti}$ & 0.557 & 0.799 & -0.030 & 0.129 \\
\hline $\mathrm{V}$ & 0.557 & 0.867 & -0.032 & 0.174 \\
\hline $\mathrm{Cr}$ & 0.423 & 0.604 & -0.065 & -0.346 \\
\hline $\mathrm{Mn}$ & 0.093 & 0.898 & 0.010 & 0.190 \\
\hline $\mathrm{Fe}$ & 0.306 & 0.840 & 0.005 & 0.146 \\
\hline $\mathrm{Ni}$ & 0.459 & 0.640 & -0.034 & -0.322 \\
\hline $\mathrm{Co}$ & -0.018 & 0.931 & -0.020 & 0.034 \\
\hline $\mathrm{Cu}$ & 0.248 & 0.549 & 0.578 & 0.109 \\
\hline $\mathrm{Zn}$ & 0.120 & 0.016 & 0.972 & -0.030 \\
\hline $\mathrm{Se}$ & 0.037 & -0.179 & 0.864 & 0.415 \\
\hline As & 0.527 & 0.306 & 0.183 & 0.744 \\
\hline $\mathrm{Br}$ & 0.133 & 0.127 & 0.302 & -0.073 \\
\hline $\mathrm{Rb}$ & 0.944 & 0.139 & 0.049 & 0.234 \\
\hline $\mathrm{Sr}$ & 0.328 & 0.442 & 0.566 & -0.007 \\
\hline $\mathrm{Zr}$ & 0.929 & 0.327 & 0.012 & 0.069 \\
\hline Mo & 0.532 & 0.100 & 0.096 & 0.779 \\
\hline $\mathrm{Ag}$ & -0.053 & -0.159 & 0.969 & -0.029 \\
\hline $\mathrm{Cd}$ & 0.073 & 0.139 & 0.024 & 0.031 \\
\hline In & 0.150 & 0.436 & 0.379 & -0.350 \\
\hline $\mathrm{Sb}$ & 0.685 & 0.419 & 0.236 & 0.435 \\
\hline I & -0.085 & -0.313 & 0.335 & -0.035 \\
\hline $\mathrm{Ba}$ & 0.882 & 0.348 & 0.049 & 0.077 \\
\hline Cs & 0.806 & 0.149 & 0.132 & 0.487 \\
\hline $\mathrm{La}$ & 0.840 & 0.336 & -0.046 & 0.392 \\
\hline $\mathrm{Ce}$ & 0.845 & 0.361 & -0.028 & 0.352 \\
\hline $\mathrm{Nd}$ & 0.909 & 0.262 & -0.073 & 0.213 \\
\hline $\mathrm{Sm}$ & 0.764 & 0.423 & -0.080 & 0.377 \\
\hline $\mathrm{Eu}$ & 0.687 & 0.540 & -0.041 & 0.375 \\
\hline $\mathrm{Gd}$ & 0.873 & 0.362 & -0.038 & -0.171 \\
\hline $\mathrm{Tb}$ & 0.556 & 0.639 & -0.044 & 0.387 \\
\hline Dy & 0.561 & 0.592 & 0.048 & 0.371 \\
\hline $\mathrm{Tm}$ & 0.561 & 0.662 & -0.032 & 0.216 \\
\hline $\mathrm{Yb}$ & 0.591 & 0.667 & 0.074 & 0.172 \\
\hline $\mathrm{Lu}$ & -0.179 & -0.354 & -0.221 & -0.092 \\
\hline Hf & 0.905 & 0.391 & 0.000 & 0.033 \\
\hline $\mathrm{Ta}$ & 0.822 & 0.489 & 0.122 & -0.059 \\
\hline W & 0.073 & -0.075 & -0.283 & -0.141 \\
\hline $\mathrm{Au}$ & 0.488 & 0.175 & 0.146 & 0.738 \\
\hline $\mathrm{Hg}$ & 0.423 & 0.326 & 0.024 & 0.382 \\
\hline $\mathrm{Th}$ & 0.887 & 0.217 & 0.379 & 0.068 \\
\hline $\mathrm{U}$ & 0.900 & 0.195 & 0.236 & 0.043 \\
\hline Variance $(\%)$ & 55.85 & 10.9 & 9.49 & 7.6 \\
\hline
\end{tabular}


Fig. 2 SEM photos of earthorigin (objects 1-6) and spaceorigin (objects 6-12)

microparticles
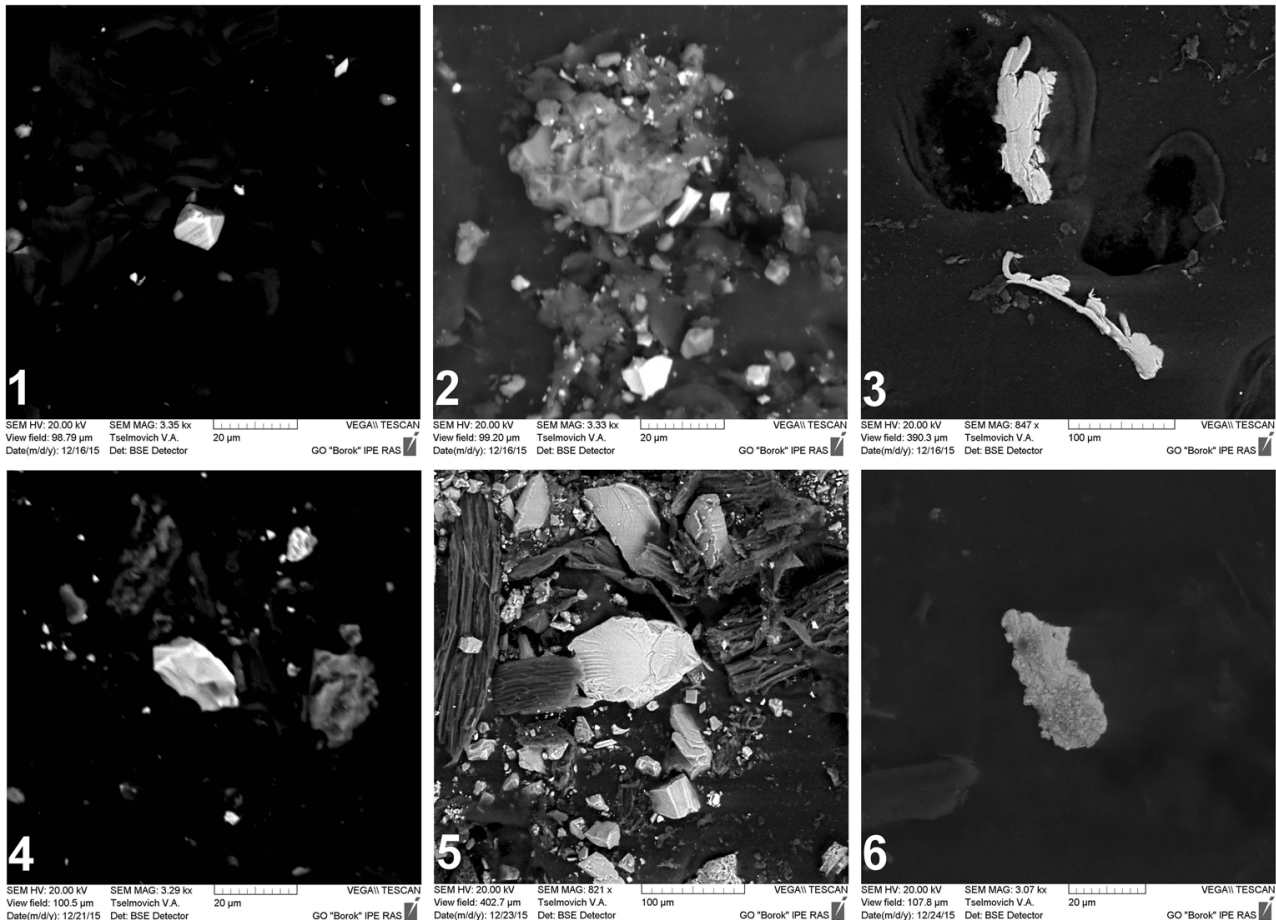

co "Borok' PE RAS
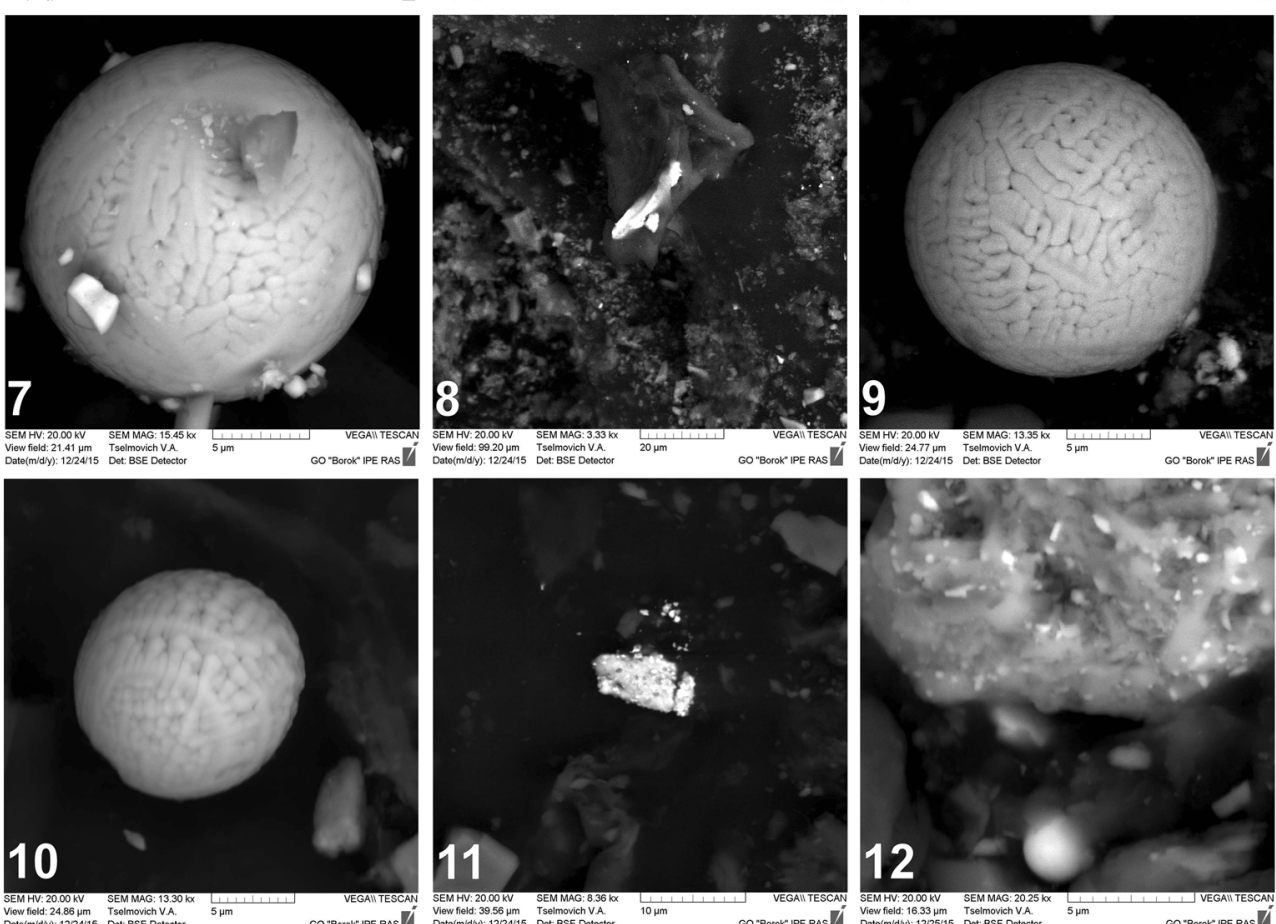

arsenopyrite. The natural background can be also elevated due to volcanic activity (Sieprawska et al. 2015; Schwanck et al. 2016). On the other hand, Amouroux et al. (2001) described mechanism of releasing Se from oceans. In effect of biogenic activity, volatile compounds as dimethyl sulfide (DMS) and dimethyl selenide (DMSe) can be released from the ocean and later be deposited on a land surface. Oceans can be also source of Cd (Bargagli et al. 1998). For some element deposition, also local microclimate can be important and must be taken into account (Zvěrina et al. 2014). For establishing potential sources of pollution, statistical methods can be used. In our work, factor analysis was used. Based on this approach, four factors can be distinguished as an origin of pollutants in lichen and moss samples.

Factor 1 represents typical crustal composition and is associated with resuspension of soil and rock particles. Factor 2 
can be probably associated with volcanic activity. Antarctic Peninsula has extensive volcanic system, and Deception Island is one of active volcanoes. Soils of King George Island have high content of tephra and ash (Deheyn et al. 2005), which probably are resuspended similar to factor 1 . Factors 3 and 4 are probably connected with human activity on island and long-range transport of pollutants. There is also a possibility that some of pollutants in these factors are delivered by birds eating contaminated fishes. Pollutants could be then deposited on island with feces. That situation can be observed near Admiralty Bay where penguin colonies and ornithogenic soils are present, and arsenic concentrations were in general higher than in other sampling sites, and arsenic enriches in penguin dropping sediment (Xie and Sun 2008). Factor 4 includes Mo and As. Both of them can be local pollutants or transported to the island (Schwanck et al. 2016), where Mo may be reduced to insoluble form by bacterial activity (Ahmad et al. 2013). Chromium and Ni can be connected with combustion of diesel oil or with construction materials like stainless steel (Barałkiewicz and Siepak 1999; Kotaś and Stasicka 1999).

\section{SEM results}

SEM microscopy images of investigated samples of moss and lichen showed presence of volcano and cosmic-originated particles in all samples (Fig. 2). First six samples (1-6) are probably volcanic-originated. Sample 1 is titanomagnetite monocrystal. Number 2 is also titanomagnetite but with detrital structure. Number 3 is a native $\mathrm{Al}$, with $5 \% \mathrm{Mg}$ impurity. Sample number 4 is a pyrite (Fe $45 \%, \mathrm{~S} 48 \%$, and $\mathrm{O} 7 \%$ by mass). Next sample (number 5) is a pyroxene and titanomagnetite. Large object in center of image is a pyroxene (44\% O, 9\% Mg, 2\% Al, 23\% Si, 13\% Ca, and $10 \% \mathrm{Fe}$ by mass), and small bright spots are titanomagnetites (35\% O, $2 \% \mathrm{Al}, 1 \% \mathrm{Si}, 6 \% \mathrm{Ti}$, and $56 \% \mathrm{Fe}$ ); sample 6 is native iron. Samples 7, 9, 10, and 12 are spherical object space origin classified due to their dimensions as IDP and with chemical composition of Fe and O, without Ti. Samples 8 and 11 have differed chemical composition. In sample 8 , we have $6 \% \mathrm{O}$, $16 \% \mathrm{Cr}$, and $78 \%$ of $\mathrm{Fe}$ and its chemical composition can be evidence for cosmic origin (Korchagin et al. 2010). Finally, sample 11 is made from $\mathrm{Ti}(2 \%), \mathrm{Ni}(4 \%), \mathrm{Cu}(49 \%), \mathrm{Zn}$ $(17 \%)$, and $\mathrm{W}(28 \%)$ and is a rare example of cosmic particle with $\mathrm{Cu}-\mathrm{Zn}$ alloy (Korchagin et al. 2010) where Ti can be contaminated from basalts (Pechersky et al. 2015a, b). The spherical IDPs found in our samples are type I microspherules (Brownlee et al. 1997) and are composed mainly from $\mathrm{Fe}_{3} \mathrm{O}_{4}$ and $\mathrm{FeO}$ (Engrand et al. 2005). As the average flux of cosmic dust is $1 / \mathrm{m}^{2} / \mathrm{d}$ for $10 \mu \mathrm{m}$ particles and $1 / \mathrm{m}^{2} / \mathrm{y}$ for $100 \mu \mathrm{m}$ (McDonnell 1978; McDonnell et al. 1984), total annual deposition of cosmic particles on King George Island $\left(1150 \mathrm{~km}^{2}\right)$ can be estimated as $4.2 \cdot 10^{11} 10 \mu \mathrm{m}$ particles and $1.15 \cdot 10^{9}$ $100 \mu \mathrm{m}$ particles.

\section{Summary}

In this study, 50 element concentrations were determined using instrumental neutron activation analysis in moss and lichen samples. Based on INAA and statistical results (PCA analysis), a few possible sources of pollution of King George Island were suggested. Also, presence of extraterrestrial material in investigated samples was confirmed by SEM microscopy analysis.

Acknowledgements We thank staff of Neutron Activation Analysis Sector of Frank Laboratory of Neutron Physics for helping in sample preparing, irradiation, and measurements.

Open Access This article is distributed under the terms of the Creative Commons Attribution 4.0 International License (http:// creativecommons.org/licenses/by/4.0/), which permits unrestricted use, distribution, and reproduction in any medium, provided you give appropriate credit to the original author(s) and the source, provide a link to the Creative Commons license, and indicate if changes were made.

\section{References}

Ahmad SA, Shukor MY, Shamaan NA, Mac Cormac WP, Syed MA (2013) Molybdate reduction to molybdenum blue by an Antarctic bacterium. Biomed Res Int 2013:871941

Allajbeu S, Yushin N, Qarri F, Duliu O, Lazo P, Frontasyeva MV (2016) Atmospheric deposition of rare earth elements in Albania studied by the moss biomonitoring technique, neutron activation analysis and GIS technology. Environ Sci Pollut Res Int 23:14087-14101

Amaro E, Padeiro A, Mão de Ferro A, Mota AM, Leppe M, Verkulich S, Hughes KA, Peter HU, Cánario J (2015) Assessing trace element contamination in Fildes peninsula (king George Island) and Ardley Island, Antarctic. Mar Pollut Bull 97:523-527

Amouroux D, Liss PS, Tessier E, Hamren-Larsson M, Donard OFX (2001) Role of oceans as biogenic sources of selenium. Earth Planet Sci Lett 5878:1-7

Aubert D, LeRoux G, Krachler M, Cheburkin A, Kober B, Shotyk W, Stille P (2006) Origin and fluxes of atmospheric REE entering an ombrotrophic peat bog in Black Forest (SW Germany): evidence from snow lichens and mosses. Geochim Cosmochim Acta 70: 2815-2826

Barałkiewicz D, Siepak J (1999) Chromium, nickel and cobalt in environmental samples and existing legal norms. Pol J Environ Stud 8: 201-208

Barandovski L, Cekova M, Frontasyeva M V, Pavlov S S, Stafilov T, Steinnes E, Urumov V (2006) Air pollution studies in Macedonia using the moss biomonitoring technique, NAA, AAS and GIS technology. Preprint JINR E18-2006-160, Dubna

Bargagli R (2008) Environmental contamination in Antarctic ecosystems. Sci Total Env 400:212-226

Bargagli R, Sanchez-Hernandez JC, Martella L, Monaci F (1998) Mercury, cadmium and lead accumulation in Antarctic mosses growing along nutrient and moisture gradients. Polar Biol 19:316322 
Bargagli R, Sanchez-Hernandez JC, Monaci F (1999) Baseline concentrations of the elements in the Antarctic macrolichen Umbilicaria decussata. Chemosphere 38:475-487

Bargagli R, Forghini F, Celesti C (2000) Elemental composition of the lichen Umbilicaria decussata. Ital J Zool 67:157-162

Bode P (1996) Instrumental and organizational aspects of a neutron activation analysis laboratory. Delft University of Technology, Interfaculty Reactor Institute, Delft, Netherland

Bötler M (2011) Soil development and soil biology on King George Island, Maritime Antarctic. P Pol Res 30:105-116

Brioschi L, Steinmann M, Lucot E, Pierret MC, Stille P, Prunier J, Badot PM (2013) Transfer of rare earth elements (REE) from natural soil to plant systems: implications for the environmental availability of anthropogenic REE. Plant Soil 366:143-163

Brownlee DE (1985) Cosmic dust: collection and research. Ann Rev Planet Sci 13:147-173

Brownlee DE, Bates B, Schramm L (1997) The elemental composition of stony cosmic spherules. Meteorit Planet Sci 32:157-175

Bubach D, Perez-Catán S, Di Fonzo C, Dopchiz L, Arribére M, Ansaldo M (2016) Elemental composition of Usnea sp. Lichen from potter peninsula 25 de Mayo (King George) Island Antarctica. Environ Pollut 210:238-245

Chwedorzewska KJ, Korczak M (2010) Human impact upon the environment in the vicinity of Arctowski station, King George Island, Antarctica. P Pol Res 31:45-60

Deheyn DD, Gendreau P, Baldwin RJ, Latz MI (2005) Evidence for enhanced bioavailability of trace elements in the marine ecosystem of Deception Island, a volcano in Antarctica. Mar Environ Res 60: $1-33$

Engrand C, McKeegan KD, Leshin LA, Herzog GF, Schnabel C, Nyquist LE, Brownlee DE (2005) Isotopic compositions of oxygen, iron, chromium and nickel in cosmic spherules: toward a better comprehension of atmospheric entry heating effects. Geochim Cosmochim Acta 69:5365-5385

Frontasyeva MV (2011) Neutron activation analysis in the life sciences. Phys Part Nuclei 42:332-378

Ganeva A, Yurukova L (2004) Data on species composition and background concentrations of some elements in moss samples from Livingston Island (Antarctica). Herz 17:199-206

Genge MJ, Carleton LE, Lumpkin GR (1997) The texture and compositions of fine-grained Antarctic micrometeorites: implications for comparisons with meteorites. Geochim Cosmochim Acta 61: $5149-5162$

Gonzáles S, Osores J, Mendoza P (2002) Study of Antarctic lichens as environmental biomonitors in the Admirality Bay: preliminary study of trace elements contents by neutron activation analysis, Informe Científico-Technológico 1998-2001 del IPEN

Grachev AF, Korchagin OA, Tselmovich VA, Kollman HA (2008) Cosmic dust and micrometeorites in the transitional clay layer at the Cretaceous-Paleogene boundary in the gams section (Eastern Alps): morphology and chemical composition. Izv Phys Solid Earth 44:555-569

Gribble GW (2010) Naturally occurring organohalogen compounds - a comprehensive update. Springer Verlag/Wien, Germany

Harmens H, Mills G, Frontasyeva M V, Zinicovscaia I, Coulicov O (2016) ICP Vegetation $29^{\text {th }}$ task force meeting, February 29 March 4, 2016, Dubna, Russian Federation: programme and abstracts

Kabata-Pendias A (2000) Trace elements in soils and plants, 3rd edition. CRC Press, Taylor and Francis Group

Kejna M (1999) Air temperature on King George Island, South Shetland Islands, Antarctica. P Pol Res 20:183-201

Kłos A, Aleksiayenak YA, Ziembik Z, Rajfur M, Jerz D, Wacławek M, Frontasyeva MV (2013) The use of neutron activation analysis in the biomonitoring of trace element deposition in the Opole province. Ecol Chem Eng 20:677-687
Korchagin OA, Tselmovich VA, Pospelov II, Qiantao B (2010) Cosmic magnetite microspherules and metallic particles near the Permiantriassic boundary in a global stratotype section and point (Stratum 27, Meishan, China). Dokl Earth Sci 432:631-637

Korzewka S, Pantrakova YS, Frontasyeva MV (2007) Air pollution studies in Opole region, Poland, using the moss biomonitoring technique and neutron activation analysis. Ecol Chem Eng 1:43-51

Kotaś J, Stasicka Z (1999) Chromium occurrence in the environment and methods of its speciation. Environ Pollut 107:263-283

Lim HS, Han MJ, Seo DC, Kim JH, Lee JI, Park H, Jae-Seun H, Cheong YH, Heo JS, Yoon HI, Cho JS (2009) Heavy metal concentrations in the fructoise lichen Usnea aurantarctico-atra from King George Island, South Shetland Islands, West Antarctica. J Korean Soc Appl Biol Chem 52:503-508

Mão de Ferro A, Mota AM, Canário J (2013) Sources and transport of as, $\mathrm{cu}, \mathrm{cd}$ and $\mathrm{Pb}$ in the environmental compartments of Deception Island, Antarctica. Mar Pollut Bull 77:341-348

Markert B (1991) Estabilishing of 'reference plant' for inorganic characterization of different plant species by chemical fingerprinting. Water Air Soil Pollut 64:533-538

Matschullat J, Scharnweber T, Grabe-Schönberg D, Walther A, Wirth V (1999) Epilithic lichen-atmospheric deposition monitors of trace elements and organohalogens? J Air Waste Manag Assoc 49: 1201-1211

Maurette M, Jehanno C, Robin E, Hammer C (1986) Characteristics and mass distribution of extraterrestrial dust from the Greenland ice cap. Nature 328:699-702

McDonnell JAM (1978) Cosmic dust. Wiley, New York

McDonnell JAM, Carey WC, Dixon DG (1984) Cosmic dust collection by capture cells. Nature 309:237-240

McLennan SM, Hemming S, McDaniel DK, Hanson GN (1993) Geochemical approaches to sedimentation, provenance and tectonics. Geol Soc Am Spec Pap 284:21-40

Murray S, Renard A F (1891) Report on deep-sea deposits based on the species collected during the voyage of H.M.S challenger in the years 1873-1876. Report on the scientific results of the voyage of H.M.S. challenger during the years 1873-76; John Menzies and Co., Endinburgh, United Kingdom

Nash TH (1996) Lichen biology. Cambridge University Press, Cambridge, United Kingdom

Ostrovnaya T M (2000) Tables for identification of nuclides formed in nuclear reactors. Preprint JINR, E14-2000-178, Dubna

Ostrovnaya T M, Nefedyeva L S, Nazarov V M, Borzakov S B, Strelkova L P (1993) Software for INAA on the basis of relative and absolute methods using nuclear database in activation analysis in environment protection. Preprint JINR, D-14-93-325, Dubna

Osyczka P, Dudtkiewicz EM, Olech M (2007) Trace element concentrations in selected moss and lichen species collected within Antarctic research stations. Pol J Ecol 55:39-48

Pechersky DM, Kuzina DM, Nurgaliev DK, Tselmovich VA (2015a) The common nature of native iron in terrestrial rocks and meteorites: microprobe and thermomagnetic data. Izv Phys Solid Earth 51: 748-763

Pechersky PM, Markov GP, Tselmovich VA (2015b) Pure iron and other magnetic minerals in meteorites. Sol Syst Res 49:61-71

Putzke J, Athanásio CG, Margéli P, Victoria FC, Pereira AB (2015) Comparative study of moss diversity in South Shetland Islands and in Antarctic Peninsula. Rev Chil Hist Nat 88:1-6

Rudnick RL, Gao S (2004) Composition of the continental crust. Treatise on Geochemistry 3:1-64

Ryghaug P (1983) Geochemical exploration methods for nobium and rare earth elements at Sætcråscn. Vestfold volcanic area, Oslo graben. Nor Geol Tidsskr 63:1-13

Schwanck F, Simoes JC, Handley M, Mayewski PA, Bernardo RT, Aquino FE (2016) Anomalously high arsenic concentration in a 
West Antarctic ice core and its relationship to copper mining in Chile. Atmos Environ 125:257-264

Shaw GE (1988) Antarctic aerosols - a review. Rev Geophys 26:89-112 Sieprawska A, Kornas A, Filek M (2015) Involvement of selenium in protective mechanisms of plants under environmental stress condition-review. Acta Biol Cracov Ser Bot 57:9-20

Sloof JE (1993) Environmental lichenology: biomonitoring trace element air pollution. Delft University of Technology, Interfaculty Reactor Institute, Delft, Netherland, Doctor's thesis

Smykla J, Szarek-Gwiazda E, Krzewicka B (2005) Trace elements in the lichens Usnea aurantarctico-atra from the vicinity of Uruguay's Artigas research station on King George Island Maritime Antarctic. Pol Bot Stud 19:49-57

Steiness E (1995) A critical evaluation of the use of naturally growing moss to monitor the deposition of atmospheric metals. Sci Total Env 160:243-249

Steinnes E (2005) Values from Pritine regions of Northern Norway in 2005. Unpublished

Steinnes E, Berg T, Uggerund H, Vadset M (2007) Atmospheric deposition of heavy metals in Norway. Nation-wide survey in 2005. State program for pollution monitoring, report 980/2007, Norwegian state pollution control authority, Oslo, Norway

Szczepaniak K, Biziuk M (2003) Aspects of the biomonitoring studies using mosses and lichens as indicators of metal pollution. Env Res 93:221-230

Szöőr G, Elekes Z, Rózsa UI, Simulák J, Kiss ÁZ (2001) Magnetic spherules: cosmic dust or markers of meteoritic impact? Nucl Instr Meth Phys Res B 181:557-562

Tatur A, Myrcha A (1984) Ornithogenic soils on King George Island, South Shetland Islands (Maritime Antarctic Zone). P Pol Res 5:3160

Taylor SR, McLennan SM (1985) The continental crust: its composition and evolution. Blackwell Scientific Publications, Oxford, United Kingdom
Taylor SR, McLennan SM (1995) The geochemical evolution of the continental crust. Rev Geophys 33(2):241

Thinova L, Frontasyeva MV, Vergel K, Bayushkina E (2014) Assessment of contamination with trace elements and man-made radionuclides around Temelin nuclear power plant in Czech Republic. Radiat Phys Chem 104:432-435

Turetsky MR, Bond-Lamberty B, Talbot J, Frolking S, McGuire AD, Tuittila ES (2012) The resilience and functional role of moss in boreal and artcic ecoystems. New Phytol 196:49-67

Victoria FDC, Pereira AB, da Costa DP (2009) Composition and distribution of moss formations in the ice-free areas adjoining the Arctowski region, Admirality Bay, King George Island, Antarctica. IHERINGA, Sér Bot 64:81-91

Victoria FDC, Margéli P, Pereira AB, Simas FN, Spielmann AA, Schaefer CE (2013) Characterization and mapping of plant communities at Hennequin Point, King George Island, Antarctica. Polar Res 32:19261

Wu Q, Wang X, Zhou Q (2014) Biomonitoring persistent organic pollution in the atmosphere with mosses: performance and application. Environ Int 66:28-37

Xie Z, Sun L (2008) A 1,800-year record of arsenic concentration in the penguin dropping sediment, Antarctic. Environ Geol 55:1055-1059

Yada T, Nakamura T, Takaoka N, Noguchi T, Terada K, Yano H, Nakazawa T, Kojima H (2004) The global accretion rate of extraterrestrial materials in the last glacial period estimated from the abundance of micrometeorites in antarctic glacier ice. Earth Planets Space 56:67-79

Zvěrina O, Láska K, Červernka R, Kuta J, Coufalík P, Komárek J (2014) Analysis of mercury and other heavy metals accumulated in lichen Usnea antarctica from James Ross Island, Antarctica. Environ Monit Assess 186:9089-9100 